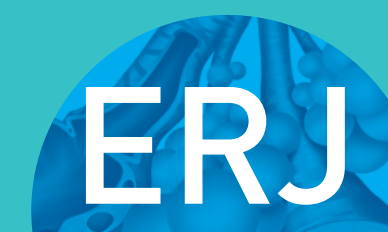

open research
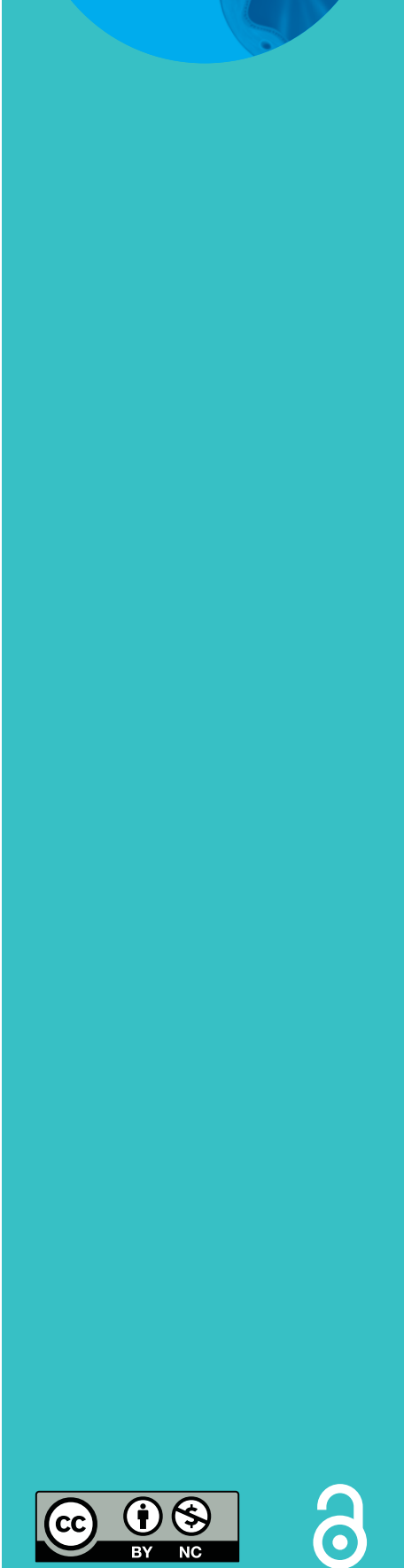

\section{Mask pressure effects on the nasal bridge during short-term noninvasive ventilation}

\author{
Anne-Kathrin Brill ${ }^{1,2}$, Rachel Pickersgill ${ }^{1}$, Mohammad Moghal ${ }^{1,3}$, \\ Mary J. Morrell ${ }^{1,3}$ and Anita K. Simonds ${ }^{1,3}$
}

Affiliations: ${ }^{1}$ Academic Unit of Sleep and Breathing, National Heart and Lung Institute, Imperial College, London, UK. ${ }^{2}$ Dept of Pulmonary Medicine, University and University Hospital Bern, Bern, Switzerland. ${ }^{3}$ National Institute for Health Research (NIHR) Respiratory Biomedical Research Unit, Royal Brompton and Harefield Foundation Trust and Imperial College, London, UK.

Correspondence: Anne-Kathrin Brill, University Hospital and University of Bern, Department of Pulmonary Medicine, 3010 Bern, Switzerland. E-mail: anne-kathrin.brilldinsel.ch

ABSTRACT The aim of this study was to assess the influence of different masks, ventilator settings and body positions on the pressure exerted on the nasal bridge by the mask and subjective comfort during noninvasive ventilation (NIV).

We measured the pressure over the nasal bridge in 20 healthy participants receiving NIV via four different NIV masks (three oronasal masks, one nasal mask) at three different ventilator settings and in the seated or supine position. Objective pressure measurements were obtained with an I-Scan pressuremapping system. Subjective comfort of the mask fit was assessed with a visual analogue scale.

The masks exerted mean pressures between $47.6 \pm 29 \mathrm{mmHg}$ and $91.9 \pm 42.4 \mathrm{mmHg}$ on the nasal bridge. In the supine position, the pressure was lower in all masks $(57.1 \pm 31.9 \mathrm{mmHg}$ supine, $63.9 \pm 37.3 \mathrm{mmHg}$ seated; $\mathrm{p}<0.001$ ). With oronasal masks, a change of inspiratory positive airway pressure (IPAP) did not influence the objective pressure over the nasal bridge. Subjective discomfort was associated with higher IPAP and positively correlated with the pressure on the skin.

Objective measurement of pressure on the skin during mask fitting might be helpful for mask selection. Mask fitting in the supine position should be considered in the clinical routine.

@ERSpublications

The pressure exerted by NIV masks on the nasal bridge is lower in the supine position and not influenced by the IPAP http://ow.ly/8nkE30iI6Kd

Cite this article as: Brill A-K, Pickersgill R, Moghal M, et al. Mask pressure effects on the nasal bridge during short-term noninvasive ventilation. ERJ Open Res 2018; 4: 00168-2017 [https://doi.org/ 10.1183/23120541.00168-2017].

Received: Dec 202017 | Accepted after revision: Feb 232018

Copyright $\odot$ ERS 2018. This article is open access and distributed under the terms of the Creative Commons Attribution Non-Commercial Licence 4.0. 


\section{Introduction}

Noninvasive ventilation (NIV) is an established treatment for acute and chronic respiratory failure [1], but the adherence to NIV and its effectiveness may be reduced by mask-related side effects. Discomfort from the mask and skin breakdown, which can occur even after only a few hours of treatment, are common problems during mask ventilation and NIV masks have been identified as a relevant source of medical device-related pressure ulcers [2-5]. Despite the awareness of the problem and the increasing number of available interface types and designs, pressure sores are still a frequent complication in the acute NIV setting, affecting $10-31 \%$ of patients [3,6-13]. This can partially be attributed to the extension of NIV treatment to more severely ill or frail patients and those at the extremes of age, the establishment of high-intensity ventilator settings and the increasing use of NIV outside experienced intensive care or respiratory units [14-16].

The area of the face that is predominantly affected by pressure effects from NIV is the nasal bridge $[1,2]$. This can be explained by anatomical factors including reduced tissue perfusion over the bony prominence of the nose and the usually higher pressures exerted by oronasal and nasal NIV masks on this facial region [17-21].

An appropriate choice of the interface and a good mask fit can influence the pressure exerted by NIV masks over the nasal bridge [22], but overall there is little information on the pathophysiology of facial pressure necrosis induced by facemasks and factors that might influence the created pressure.

This study aimed to objectively quantify the pressure exerted by NIV masks on the skin over the nasal bridge in vivo. We hypothesised that different body positions, ventilator settings and mask designs can significantly change the pressure exerted on the skin by the NIV mask and also affect the users' comfort level.

\section{Material and methods}

The study was conducted in the in the National Institute for Health Research (NIHR) Respiratory Biomedical Research Unit of the Royal Brompton and Harefield NHS Foundation Trust (London, UK) from June 2014 until January 2015. The study was approved by the London-Riverside Research Ethics Committee (REC number: 14/LO/0809), and was conducted according to the ethical principles of the Declaration of Helsinki. All participants gave written informed consent prior to inclusion in the study.

\section{Study participants}

Eligible healthy volunteers naïve to NIV were recruited by word of mouth. Inclusion criteria were an age of 18 years or older and the ability to consent. Exclusion criteria were pre-existing lesions or a rash on the face in the area of mask application, pregnancy, current or chronic respiratory disease or nasal symptoms, and a history of claustrophobia or pneumothorax.

\section{Experimental protocol, masks, ventilator settings and postures}

We investigated four different commercially available vented NIV masks: a disposable oronasal mask with a dual wall cushion (Hospital Fullface; ResMed Ltd, Bella Vista, Australia), a reusable oronasal mask with a dual wall cushion (Quattro Air; ResMed Ltd), a reusable oronasal mask with a gel cushion and additional silicon flap (Comfort Gel Full; Philips Respironics Inc., Murrysville, PA, USA), and a reusable nasal mask with a dual silicone cushion construction (Easylife; Philips Respironics Inc., Murrysville, PA, USA). For all measurements a Stellar 150 ventilator (ResMed Ltd) was used in a flow-triggered spontaneous breathing mode (S-mode) with a single limb circuit and without a humidifier.

The four NIV masks were applied randomly at the following different ventilator settings and body positions in each participant: inspiratory positive airway pressures (IPAP) of 15,20 or $25 \mathrm{~cm} \mathrm{H}_{2} \mathrm{O}$ with the volunteer being seated, and in the supine position with an IPAP of $20 \mathrm{~cm} \mathrm{H}_{2} \mathrm{O}$. The expiratory positive airway pressure was maintained at $5 \mathrm{~cm} \mathrm{H}_{2} \mathrm{O}$ for all measurements. The mask types, pressure settings and postures were studied in a random order on each participant.

\section{Mask fitting and pressure measurement}

Mask fittings and pressure measurements were performed in a standardised way by two investigators who were experienced in NIV (A-K. Brill; R. Pickersgill). The mask sizes were chosen individually for each participant according to the manufacturers' instructions with the provided sizing gauges. Pressure measurements were taken over the nasal bridge as the most vulnerable part of the face with an I-Scan ${ }^{\circ}$ pressure mapping system (Tekscan Inc., South Boston, MA, USA) and a 4201-pressure sensor as described previously [22]. In brief, the sensor was calibrated with a four-point calibration with a PB5A calibration device (Tekscan Inc.). The thin and flexible sensor was placed in the same position over the nasal bridge between the facial skin and NIV mask for all measurements. The mask was gently placed over the pressure 
sensor on the participants' face and connected to the ventilator. The mask fit was then adjusted until there was no or minimal air-leak from the interface defined as an unintentional air-leak of $<10 \mathrm{~L} \cdot \mathrm{min}^{-1}$ detected by the ventilators inbuilt leak-detection programme and no irritating air-leak alongside the mask disturbing the participant. After completion of the mask fitting we allowed for a 5-8 min equilibrium period of stable breathing on NIV. Leaks that occurred during the equilibrium interval and exceeded $10 \mathrm{~L} \cdot \mathrm{min}^{-1}$ were corrected. Then, the contact pressure exerted by the NIV mask was recorded. To account for pressure changes during the respiratory cycle, the outcome parameter for the pressure measurements was defined as the mean contact pressure over the $2.5 \mathrm{~cm}^{2}$ measurement area over the nasal bridge during a measurement period of $25 \mathrm{~s}$. After each measurement, NIV was stopped, the mask removed and refitted for the next measurement. The average duration of assessment time for all pressure settings and postures was about $1 \mathrm{~h}$ per mask. The facial skin in the area of the mask and pressure sensor placement was assessed before and after the completion of the session.

\section{Subjective comfort}

Subjectively perceived comfort of the mask fit was assessed using a handhold $100 \mathrm{~mm}$ long visual analogue scale (VAS) for mask discomfort ranging from $0-100$, with 0 being most comfortable and 100 being least comfortable $[9,22,23]$. Subjective comfort was rated immediately after each pressure measurement. The participants were blinded to the results of the pressure measurement over the nasal bridge.

\section{Statistical analysis}

Data are presented as mean \pm SD. Differences between ventilator settings and masks were assessed with repeated measurements ANOVA and post hoc t-tests with a Bonferroni correction for pairwise comparisons. The two postures were compared for each mask with paired t-tests or Wilcoxon signed rank tests depending on normality distribution. Pearson's correlation coefficient was used to assess the relationship between variables. The significance level of all analyses was set to 0.05 . Statistical analysis was performed using SPSS version 22 (IBM, Chicago, IL, USA) and Graphpad Prism 6 (Graphpad software, La Jolla, CA, USA).

\section{Results}

Study participants

All 20 participants (12 male, eight female) completed the measurements with valid data. Participants were healthy with a mean age of $36 \pm 11$ years and BMI of $25.1 \pm 3.3 \mathrm{~kg} \cdot \mathrm{m}^{-2}$. The self-reported ethnicity of the participants was predominantly Caucasian (14 Caucasian, one Chinese, one African, and four Asian other). No-one used analgesic medication.

\section{IPAP levels}

Repeated measures ANOVA showed that the mean contact pressure over the nasal bridge did not differ between the different IPAP levels with the three oronasal masks while with the nasal mask there was a higher nasal bridge contact pressure of $5.6 \pm 5.8 \mathrm{mmHg}, \mathrm{p}<0.001$ at an IPAP of $25 \mathrm{~cm} \mathrm{H}_{2} \mathrm{O}$ compared with an IPAP of $15 \mathrm{~cm} \mathrm{H}_{2} \mathrm{O}$ (table 1 and figure 1). There were no statistically significant differences in air-leak between the different IPAP levels for all masks, which can be attributed to the study design in which mask leak was assessed systematically and a fine-tuning of mask fit carried out until the leak was minimised $<10 \mathrm{~L} \cdot \mathrm{min}^{-1}$.

Subjective comfort levels showed an increase towards more discomfort on the VAS with higher IPAP levels with the three oronasal masks and no significant change with the nasal mask. Overall, there was a weak positive correlation between higher nasal bridge contact pressure and mask discomfort with all masks (correlation coefficient $\mathrm{r}=0.247, \mathrm{n}=320 ; \mathrm{p}<0.001$ ).

\section{Body position}

The contact pressure over the nasal bridge was significantly lower in the supine position compared with the seated position in all masks with an IPAP of $20 \mathrm{~cm} \mathrm{H}_{2} \mathrm{O}$ and EPAP $5 \mathrm{~cm} \mathrm{H}_{2} \mathrm{O}$. Overall the pressure was $57.1 \pm 31.9 \mathrm{mmHg}$ supine and $63.9 \pm 37.3 \mathrm{mmHg}$ seated $(\mathrm{p}<0.0001)$. Values for the individual masks with significant reductions of the pressure on the skin in the supine position ranging between $4.1 \pm 6.9 \mathrm{mmHg}$ (nasal mask Easy life) to $12.8 \pm 11.9 \mathrm{mmHg}$ (oronasal mask Comfort Gel) are shown in table 2. Subjectively perceived comfort of the mask fit was not influenced by the posture (table 2).

\section{Mask designs}

The repeated measures ANOVA showed significant differences in nasal bridge contact pressure (F (1.942; 228)=93.698; $\mathrm{p}<0.001$ ) across the tested masks, irrespective of the ventilator setting or body position of the participant. The oronasal mask Hospital Fullface created the lowest pressures followed by the Easy life 
TABLE 1 Comparison of different inspiratory positive airway pressure (IPAP) levels in a seated position in 20 participants

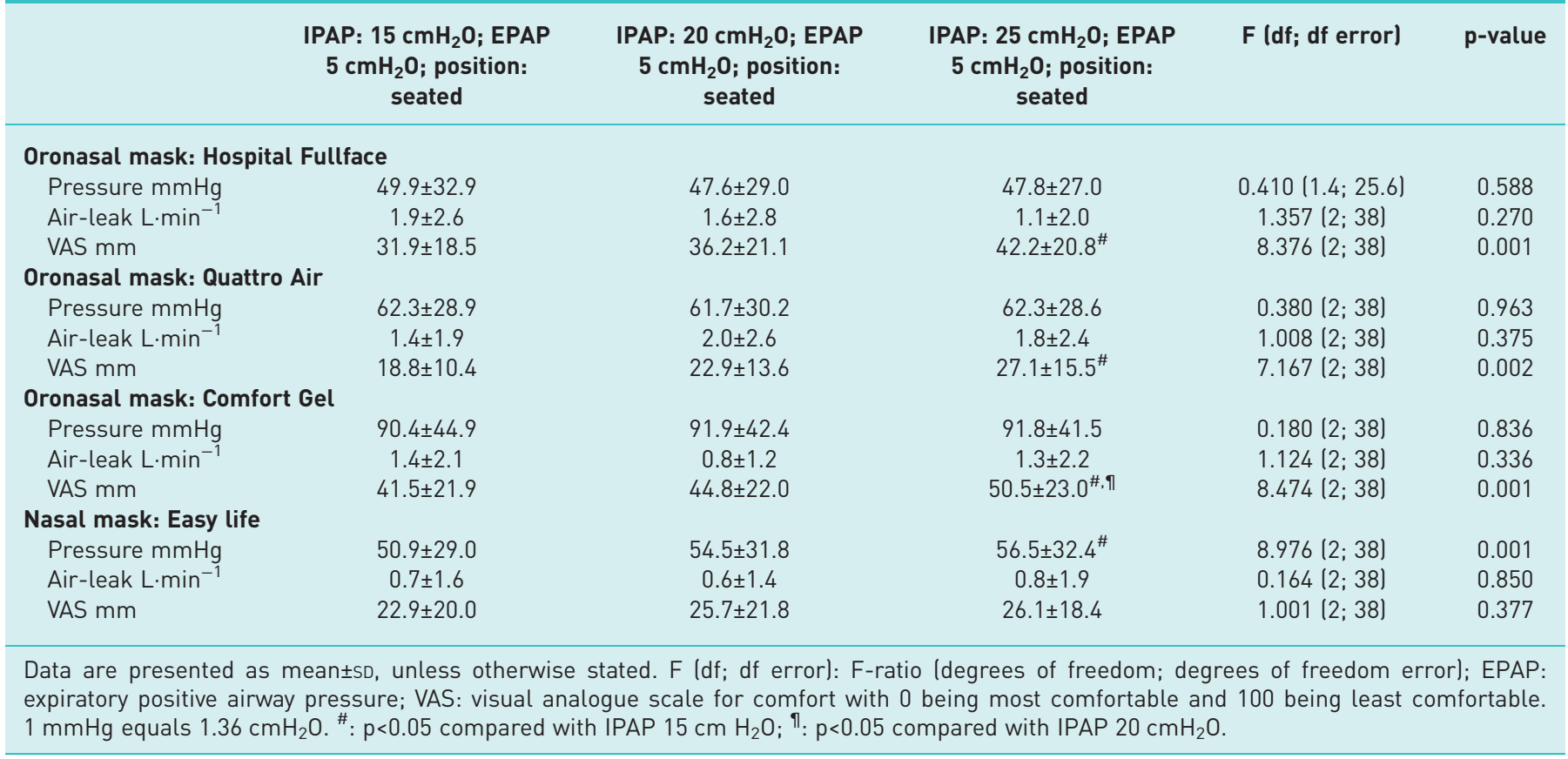

nasal mask, the Quattro Air and the Comfort Gel mask. The unintentional air-leak did not differ significantly between masks, ventilator settings and postures $(\mathrm{F}(9 ; 228)=0.72 ; \mathrm{p}=0.690)$. Subjective comfort on the VAS varied between the masks $(\mathrm{F}(7.748 ; 196.288)=0.338 ; \mathrm{p}<0.001)$ with the masks Quattro Air and Easy life being rated as most comfortable. Their ratings did not differ in the post hoc test using a Bonferroni correction, while the other two masks had significantly higher values on the VAS scale reflecting a subjectively less comfortable mask fit (tables 1 and 2).

\section{Adverse events}

No adverse events (reddening of the skin, skin lesions, pain and respiratory symptoms) occurred during the study, although exposure time was limited to the duration of the assessments.

\section{Discussion}

The main findings of this study were that an increase in IPAP does not influence the mask pressure exerted on the nasal bridge in oronasal masks, while the pressure exerted by the mask over this area is lower in the supine position than seated. We also showed that the amount of pressure exerted on the skin of the same participant can vary substantially with different mask models.

Bench studies using face masks with simple cushions and different measurement approaches provide information on the minimally necessary mask pressure to prevent air-leak, which is $1-2 \mathrm{~cm} \mathrm{H}_{2} \mathrm{O}$ above the set inspiratory ventilator pressure $[24,25]$. However, these previous bench studies do not give information on the pressure exerted to the maximal points of pressure on the face in vivo which are often higher. MunCKTON et al. [17] measured the pressure exerted on the face during NIV by simple NIV mask cushions in 2007 and identified the nasal bridge as the area of the face where the highest pressure was exerted. Since then, NIV mask design has improved and better measurement systems are now available, allowing us to carry out this assessment of the pressure applied to the nasal bridge during ventilation in different situations.

Masks are often fitted when patients are seated but, with the exceptions of daytime use in the acute setting or in home mechanical ventilation, they are normally used when the patient is recumbent. In our study, the pressure over the nasal bridge was lower in the supine position with the same level of comfort. In the absence of measurements around the total skin-mask interface this leaves us to assume that the mask fit might be improved with the help of gravitational forces that allow for less tightening of the straps or, more likely, that there is a shift of the pressure towards other areas of the face. Therefore, we think that, when possible, mask fitting in the supine position should be preferred in the clinical routine. 


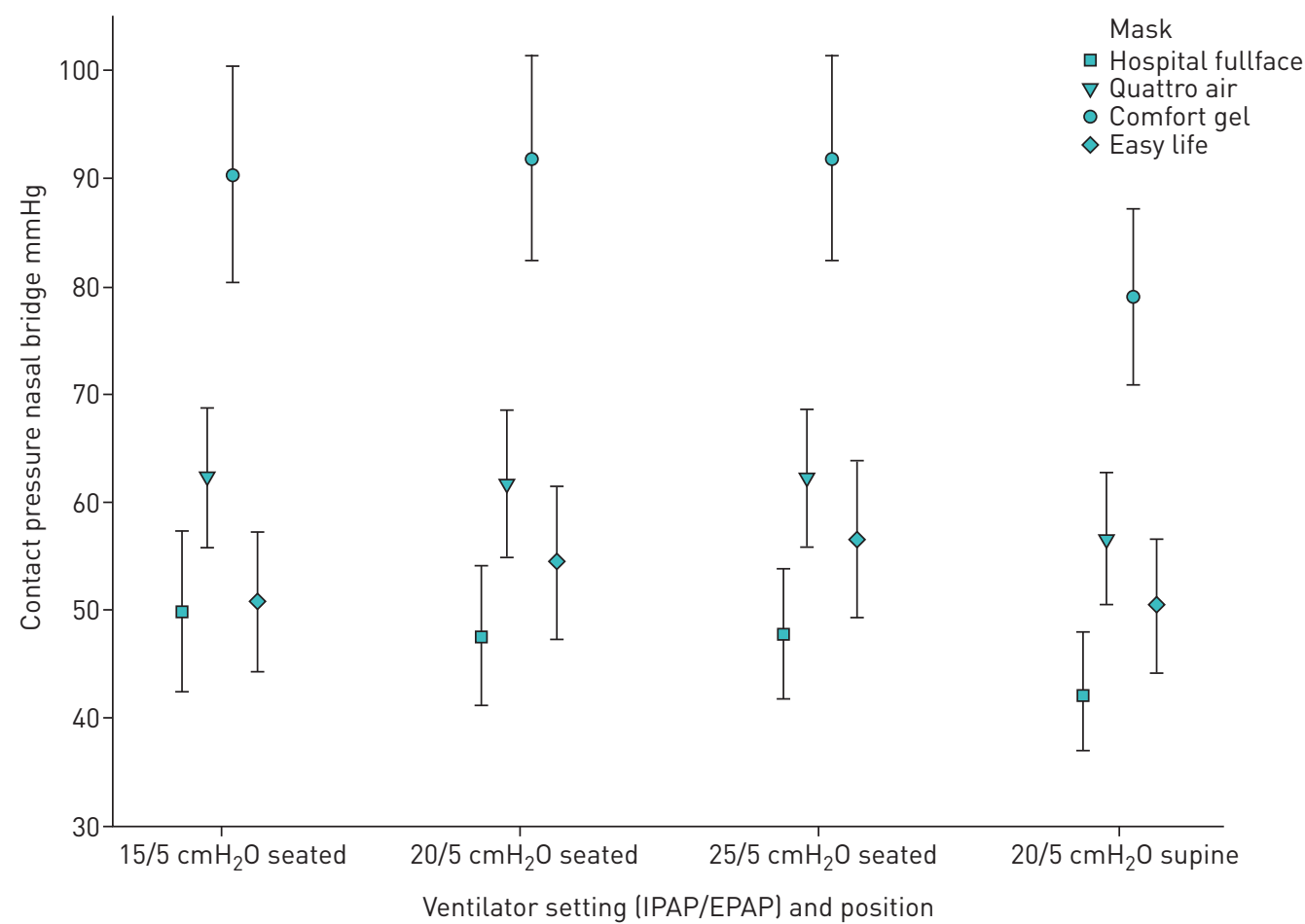

FIGURE 1 Contact pressure over the nasal bridge for the four masks and different situations. Data are presented as mean and $95 \%$ confidence interval.

It might be expected that ventilator modes that impose higher levels of respiratory pressures are associated with greater pressures from more tightly fitted masks to prevent air-leaks and therefore also have a higher risk of developing skin lesions. In this study, we demonstrated, using objective measurements that the pressure exerted on the skin over the nasal bridge does not change in appropriately fitted oronasal masks with an increase in IPAP. In addition to the already available data we showed that this is still true for

\section{TABLE 2 Comparison of two different postures in 20 participants}

IPAP: $20 \mathrm{cmH}_{2}$ O; EPAP $5 \mathrm{cmH}_{2}$; IPAP: $20 \mathrm{cmH}_{2}$ O; EPAP $5 \mathrm{cmH}_{2} 0$; p-value position: seated position: supine

$\begin{array}{lccc}\text { Oronasal mask: Hospital Fullface } & & & \\ \quad \text { Pressure } \mathrm{mmHg} & 47.6 \pm 29.0 & 42.4 \pm 24.6 & 0.017 \\ \text { Air-leak L·min } & -1 & 0.9 \pm 1.8 & 0.046 \\ \text { VAS mm } & 1.6 \pm 2.8 & 36.4 \pm 20.2 & 0.795 \\ \text { Oronasal mask: Quattro Air } & 36.2 \pm 21.1 & & \\ \quad \text { Pressure mmHg } & 61.7 \pm 30.2 & 56.6 \pm 27.2 & 0.004 \\ \quad \text { Air-leak L·min } & & 1.4 \pm 2.0 & 0.389 \\ \text { VAS mm } & 2.0 \pm 2.6 & 23.4 \pm 12.7 & 0.683 \\ \text { Oronasal mask: Comfort Gel } & 22.9 \pm 13.6 & & \\ \quad \text { Pressure mmHg } & 91.9 \pm 42.4 & 79.1 \pm 36.2 & <0.001 \\ \text { Air-leak L·min } & & 1.2 \pm 2.3 & 0.673 \\ \text { VAS mm } & 0.8 \pm 1.2 & 47.5 \pm 22.5 & 0.132 \\ \text { Nasal mask: Easy life } & 44.8 \pm 22.0 & & \\ \text { Pressure mmHg } & & 50.4 \pm 27.8 & 0.001 \\ \text { Air-leak L·min } & & 1.1 \pm 2.5 & 0.462 \\ \text { VAS mm } & 54.5 \pm 31.8 & 21.5 \pm 16.7 & 0.123\end{array}$

Data are presented as mean $\pm S D$, unless otherwise stated. IPAP: inspiratory positive airway pressure; EPAP. expiratory positive airway pressure; VAS: visual analogue scale for comfort with 0 being most comfortable and 100 being least comfortable. $1 \mathrm{mmHg}$ equals $1.36 \mathrm{cmH}_{2} \mathrm{O}$. 
oronasal masks used with an IPAP of up to $25 \mathrm{~cm} \mathrm{H}_{2} \mathrm{O}$, with an unchanged unintentional air-leak. This is important for high-intensity NIV in which oronasal masks are predominantly applied $[21,26]$.

Subjectively perceived comfort can help to determine a good mask fit, but it has been shown to be too inaccurate to determine the applied pressure over the nasal bridge [22]. The correlation of higher IPAP with more discomfort in the same mask despite unchanged objective pressures over the nasal bridge in the oronasal masks and unchanged discomfort despite higher pressures on the skin in the nasal mask prompts speculations that our NIV-naïve participants might have also focussed on other factors that influence comfort than the mask pressures only. Thus, for NIV masks, an objective measurement option to monitor and reduce pressures exerted on the face by NIV masks could be helpful to reduce pressure effects and optimise the mask fit. As a clinical comparison the estimation of endotracheal tube cuff inflation pressure by palpation is inadequate to determine an estimate of the pressure existing inside the cuff [27] and the objective monitoring of endotracheal tube cuff pressures has become a standard procedure during endotracheal intubation.

However, with the Comfort Gel mask, which applied much higher pressures than the other three models, the discomfort ratings were overall also higher than for the other masks implying that at least very high pressures are perceptible. The reason why the Comfort Gel mask created much higher pressures in our participants than the other mask models is not entirely clear. This might be due to a slightly more rigid material of the gel cushion, but we cannot exclude that the pressures would have been lower in another group of participants.

More recent mask designs and different participant characteristics may explain why the pressures created by the masks in our study were partially lower than those measured by MuNCKTON et al. [17] and in a newer in vivo mask study that reported measurements without ventilation [18]. Nevertheless, despite choosing and fitting the masks by experienced personnel and according to the manufacturers' instructions, the pressures measured over the nasal bridge still exceeded the assumed capillary closing pressures and even normal diastolic blood pressure values in some participants [28].

Pressure ulcers result from a combination of localised high pressures, friction, tissue factors as hypoxaemia, hypotension, concomitant medication such as corticosteroid therapy, and time [29-31]. Although it is advisable to keep pressures exerted on the skin by medical devices as low as possible, not every individual who is exposed to a pressure above the capillary closing pressure will develop a pressure ulcer [31]. Importantly, the exploratory design of our study does not allow conclusions on whether the finding that the IPAP level does not influence the pressure exerted to the face to a greater extend can be transferred to the risk of the development of pressure ulcers in a clinical setting over a course of days on NIV therapy, but in previous clinical studies the ventilator modes and the levels of respiratory pressure applied did not influence the occurrence of pressure ulcers once the mask was properly fitted [3, 12, 32].

In addition to short-term effects on the tissue and potential NIV failure, long-term application of masks to the face, even if used solely at night, can result in changes of facial skeletal bone formation. This has been shown in growing children using NIV [33-36] but also in adults using CPAP [37]. Similar to the short-term effects, it is not clear what the individual pressure threshold is for the inhibition of bone growth in the long-term as pressure and time are both relevant factors. Nevertheless, it is sensible that pressures exerted on the face should be kept as low as possible by providing a good mask fit.

Several issues should be considered when interpreting the findings of this study. Firstly, the short-term measurements cannot represent the pressure exerted over a longer duration of NIV but can give at least useful information on the magnitude that is applied which, in turn, might lead to a change of the mask or earlier implementation of preventive skin protective measures if the values are high. Only four interfaces were tested and the study population consisted mainly of adult Caucasians which does not allow for a generalisation of the findings to the wider population, children or indeed to other masks. Differences in nasal bridge angulations of the participants that could have influenced the pressure exerted by the masks were not assessed. We did not perform measurements in the lateral recumbent positions and thus cannot inform on the consequences on the pressures exerted on the nasal bridge in this setting. Nevertheless, this position might also be considered during mask fitting as patients will change the posture during sleep.

In conclusion, objective pressures created by masks on the vulnerable skin over nasal bridge can vary substantially with different mask models, the body position influences the pressure while an increase of IPAP does not alter the pressure to a greater extend if the mask is fitted appropriately. The implementation of objective monitoring devices into clinical routine could help to overcome the limitations of a subjective estimation of the mask pressure, help to identify safe pressure thresholds for NIV masks and thus result in more effective preventive strategies to reduce the adverse effects of interface pressure during non-invasive ventilation. 
Acknowledgement: The authors would like to thank all volunteers for their participation in this study.

Conflict of interest: A-K. Brill reports having been the recipient of a European Respiratory Society Fellowship during the conduct of the study. M.J. Morrell reports being the principal investigator on a multicentre trial for the treatment of obstructive sleep apnoea funded by ResMed.

Support statement: A-K. Brill is the recipient of a European Respiratory Society/European Lung Foundation Fellowship (LTRF 2013-1798). Funding for the equipment for this project was provided by Brompton Breathers (Royal Brompton \& Harefield Charitable Trust). Funding information for this article has been deposited with the Crossref Funder Registry.

\section{References}

1 Simonds AK. Non-invasive Respiratory Support: A Practical Handbook. 3rd Edn. Boca Raton, Hodder Arnold, 2007.

2 Schallom M, Cracchiolo L, Falker A, et al. Pressure ulcer incidence in patients wearing nasal-oral versus full-face noninvasive ventilation masks. Am J Crit Care 2015; 24: 349-356.

3 Gregoretti C, Confalonieri M, Navalesi P, et al. Evaluation of patient skin breakdown and comfort with a new face mask for non-invasive ventilation: a multi-center study. Intensive Care Med 2002; 28: 278-284.

4 Gay PC. Complications of noninvasive ventilation in acute care. Respir Care 2009; 54: 246-257.

5 Carron M, Freo U, BaHammam AS, et al. Complications of non-invasive ventilation techniques: a comprehensive qualitative review of randomized trials. Br J Anaesth 2013; 110: 896-914.

6 Benhamou D, Girault C, Faure C, et al. Nasal mask ventilation in acute respiratory failure. Experience in elderly patients. Chest 1992; 102: 912-917.

7 Vitacca M, Rubini F, Foglio K, et al. Non-invasive modalities of positive pressure ventilation improve the outcome of acute exacerbations in COLD patients. Intensive Care Med 1993; 19: 450-455.

8 Wysocki M, Tric L, Wolff MA, et al. Noninvasive pressure support ventilation in patients with acute respiratory failure. Chest 1993; 103: 907-913.

9 Kramer N, Meyer TJ, Meharg J, et al. Randomized, prospective trial of noninvasive positive pressure ventilation in acute respiratory failure. Am J Respir Crit Care Med 1995; 151: 1799-1806.

10 Wysocki M, Tric L, Wolff MA, et al. Noninvasive pressure support ventilation in patients with acute respiratory failure. A randomized comparison with conventional therapy. Chest 1995; 107: 761-768.

11 Antonelli M, Conti G, Rocco M, et al. A comparison of noninvasive positive-pressure ventilation and conventional mechanical ventilation in patients with acute respiratory failure. N Engl J Med 1998; 339: 429-435.

12 Meduri GU, Turner RE, Abou-Shala N, et al. Noninvasive positive pressure ventilation via face mask. First-line intervention in patients with acute hypercapnic and hypoxemic respiratory failure. Chest 1996; 109: 179-193.

13 Schettino G, Altobelli N, Kacmarek RM. Noninvasive positive pressure ventilation reverses acute respiratory failure in select 'do-not-intubate' patients. Crit Care Med 2005; 33: 1976-1982.

14 Schwarz SB, Magnet FS, Windisch W. Why high-intensity NPPV is favourable to low-intensity NPPV: clinical and physiological reasons. COPD 2017; 14: 389-395.

15 Scala R, Corrado A, Confalonieri M, et al. Increased number and expertise of Italian respiratory high-dependency care units: the second national survey. Respir Care 2011; 56: 1100-1107.

16 Lemyze $\mathrm{M}$, Mallat J, Nigeon $\mathrm{O}$, et al. Rescue therapy by switching to total face mask after failure of face mask-delivered noninvasive ventilation in do-not-intubate patients in acute respiratory failure. Crit Care Med 2013; 41: 481-488.

17 Munckton K, Ho KM, Dobb GJ, et al. The pressure effects of facemasks during noninvasive ventilation: a volunteer study. Anaesthesia 2007; 62: 1126-1131.

18 Worsley PR, Prudden G, Gower G, et al. Investigating the effects of strap tension during non-invasive ventilation mask application: a combined biomechanical and biomarker approach. Med Devices (Auckl) 2016; 9: 409-417.

19 Barros LS, Talaia P, Drummond M, et al. Facial pressure zones of an oronasal interface for noninvasive ventilation: a computer model analysis. J Bras Pneumol 2014; 40: 652-657.

20 Crimi C, Noto A, Princi P, et al. A European survey of noninvasive ventilation practices. Eur Respir J 2010; 36 : $362-369$

21 Callegari J, Magnet FS, Taubner S, et al. Interfaces and ventilator settings for long-term noninvasive ventilation in COPD patients. Int J Chron Obstruct Pulmon Dis 2017; 12: 1883-1889.

22 Brill AK, Moghal M, Morrell MJ, et al. Randomized crossover trial of a pressure sensing visual feedback system to improve mask fitting in noninvasive ventilation. Respirology 2017; 22: 1343-1349.

23 Aitken RC. Measurement of feelings using visual analogue scales. Proc R Soc Med 1969; 62: 989-993.

24 Schettino GP, Tucci MR, Sousa R, et al. Mask mechanics and leak dynamics during noninvasive pressure support ventilation: a bench study. Intensive Care Med 2001; 27: 1887-1891.

25 Dellweg D, Hochrainer D, Klauke M, et al. Determinants of skin contact pressure formation during non-invasive ventilation. J Biomech 2010; 43: 652-657.

26 Dreher M, Storre JH, Schmoor C, et al. High-intensity versus low-intensity non-invasive ventilation in patients with stable hypercapnic COPD: a randomised crossover trial. Thorax 2010; 65: 303-308.

27 Giusti DG, Rogari DC, Gili DA, et al. Cuff pressure monitoring by manual palpation in intubated patients: How accurate is it? A manikin simulation study. Aust Crit Care 2017; 30: 234-238.

28 Landis EM. Micro-injection studies of capillary blood pressure in human skin. Heart 1930; 15: 209-228.

29 Brill A. How to avoid interface problems in acute noninvasive ventilation. Breathe 2014; 10: 230-242.

30 Thompson D. A critical review of the literature on pressure ulcer aetiology. J Wound Care 2005; 14: 87-90.

31 Bader D, Oomens C. Recent Advances in Pressure Ulcer Research. In: Romanelli M, Clark M, Cherry G, Colin D, Defloor T, eds. Science and Practice of Pressure Ulcer Management. London, Springer London, 2006; pp. 11-26.

32 Yamaguti WP, Moderno EV, Yamashita SY, et al. Treatment-related risk factors for development of skin breakdown in subjects with acute respiratory failure undergoing noninvasive ventilation or CPAP. Respir Care 2014; 59: 1530-1536. 
33 Fauroux B, Lavis JF, Nicot F, et al. Facial side effects during noninvasive positive pressure ventilation in children Intensive Care Med 2005; 31: 965-969.

34 Villa MP, Pagani J, Ambrosio R, et al. Mid-face hypoplasia after long-term nasal ventilation. Am J Respir Crit Care Med 2002; 166: 1142-1143.

35 Roberts SD, Kapadia H, Greenlee G, et al. Midfacial and dental changes associated with nasal positive airway pressure in children with obstructive sleep apnea and craniofacial conditions. J Clin Sleep Med 2016; 12: 469-475.

36 Firouz M, Zernik J, Nanda R. Dental and orthopedic effects of high-pull headgear in treatment of Class II, division 1 malocclusion. Am J Orthod Dentofacial Orthop 1992; 102: 197-205.

37 Tsuda H, Almeida FR, Tsuda T, et al. Craniofacial changes after 2 years of nasal continuous positive airway pressure use in patients with obstructive sleep apnea. Chest 2010; 138: 870-874. 